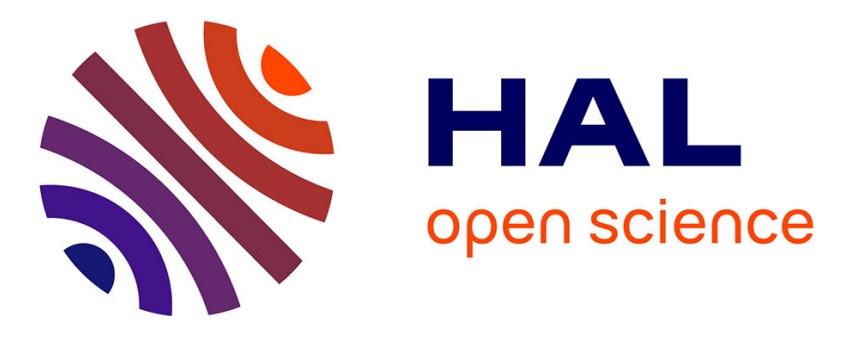

\title{
Dynamic Bayesian Networks in system reliability analysis
}

Abdeljabbar Ben Salem, Alexandre Muller, Philippe Weber

\section{To cite this version:}

Abdeljabbar Ben Salem, Alexandre Muller, Philippe Weber. Dynamic Bayesian Networks in system reliability analysis. 6th IFAC Symposium on Fault Detection, Supervision and Safety of technical processes, 2006, Beijing, China. pp.481-486. hal-00092032

\section{HAL Id: hal-00092032 \\ https://hal.science/hal-00092032}

Submitted on 8 Sep 2006

HAL is a multi-disciplinary open access archive for the deposit and dissemination of scientific research documents, whether they are published or not. The documents may come from teaching and research institutions in France or abroad, or from public or private research centers.
L'archive ouverte pluridisciplinaire HAL, est destinée au dépôt et à la diffusion de documents scientifiques de niveau recherche, publiés ou non, émanant des établissements d'enseignement et de recherche français ou étrangers, des laboratoires publics ou privés. 


\title{
DYNAMIC BAYESIAN NETWORKS IN SYSTEM RELIABILITY ANALYSIS
}

\author{
Abdeljabbar BEN SALEM ${ }^{+*}$, Alexandre MULLER ${ }^{+}$, Philippe WEBER $^{+}$ \\ ${ }^{+}$Centre de Recherche en Automatique de Nancy (CRAN - UMR 7039) Nancy-University, CNRS \\ 2, rue Jean Lamour, 54519 VANDOEUVRE-LES-NANCY Cedex - France \\ \{Philippe.Weber, Abdeljabbar.Bensalem, Alexandre.muller\}@cran.uhp-nancy.fr \\ * Institut National de recherche sur les transports et leur sécurité (INRETS) \\ 2 av Général Malleret-joinville 94114 ARCUEIL France
}

\begin{abstract}
Today industrial systems are characterized by a set of dependencies among the components and the environment of the system. To address these difficulties, this paper presents a method for modelling and analyzing the reliability of a complex system based on Dynamic Bayesian Networks (DBN). This method allows to take into account the influence of time or exogenous variables on the failure (degradation) modes of the system. The DBN graphical structure provides an easy way to specify the dependencies and, hence, to provide a compact representation of the model. In addition, the DBN formalism is associated to simulation tools that enable an efficient processing for the models. Copyright @ 2006 IFAC
\end{abstract}

Keywords: Reliability evaluation, Dynamic Bayesian Network, Markov process, Hidden Markov Model, Markov Switching Model, Input-Output HMM.

\section{INTRODUCTION}

The reliability assessment of complex systems is usually realized thanks to combinatorial methods such as Fault Tree (FT) or Reliability Block Diagram (RBD). However, these methods are not very useful to model firstly, the influence of time and exogenous variables on the degradation processes and secondly, the interdependencies among the components. Consequently, practical applications to industrial systems remain limited in number (Labeau, et al., 2000).

To make these methods more applicable, one promising solution consists in using Bayesian Networks (BN) to obtain some additional power both at the modelling and analysis levels (Bobbio, et al., 2001). BN provide a framework for handling probabilistic events and they have proven to be a powerful formalism to express complex dependencies between random variables.

Recently, their popularity started to grow among system reliability analysts. Bobbio explains, for example, how the Fault Tree (FT) can be achieved using BN. In the same way, (Torres-Toledano, et al., 2003) present the potential benefits given by Dynamic Bayesian Networks (DBN) formalism to improve the efficiency of Reliability Block Diagram (RBD). (Boudali and Dugan, 2005) describe the relation between dynamic FT and DBN. (Weber and Jouffe, 2006) propose an Object Oriented Dynamic Bayesian Network model of system reliability formalized from a dual functioning/dis-functioning modelling approach. In our previous work we have shown that DBN is an extension of Markov Chains (MC) (Weber and Jouffe, 2003), Hidden Markov Model (HMM) and Input Output Hidden Markov Model (IOHMM) (Weber et al., 2004), which decreases the effect of combinatorial explosion by a more synthetic description of a complex system. For this reason, DBN can be considered as a dynamic reliability method (Labeau, et al., 2000) capable of handling the component aging, the maintenance operations and the evolution of the environment. 
This paper introduces a modelling approach based on DBN to analyse reliability of complex systems. Thus, this study shows how DBN can be used for modelling reliability systems. Following this introduction, section 2 outlines the major concepts, proprieties and tools of the stochastic modelling of reliability. To overcome some stochastic modelling difficulties, section 3 presents the discrete-time DBN reliability framework proposed. Finally, a conclusion including some perspectives is developed in section 4 .

\section{STOCHASTIC MODEL OF SYSTEM RELIABILITY}

Representing system failure in a probabilistic way is attractive because it naturally accounts for the uncertainty and ambiguity. To make such representation, the process behaviour is considered as a random variable that takes its values from a finite state space corresponding to the possible process states.

\subsection{Time-independent Markov process}

Deterioration can be modelled by a discrete timedependent stochastic process $\left\{X_{k}, k \geq 0\right\}$ where $X_{k}$ is a random quantity for all $k \geq 0$. The reliability of systems is usually assumed to be a Markov process.

Markov process is a stochastic process evolving under the Markov property. Given a value of $X_{k}$, the values of $X_{k+i}$ where $i>0$ are independent of values of $X_{k-j}$ where $j>0$. In the case of finite or countable state space, Markov processes can be represented by a graphic called Markov Chain (Ansell, et al., 1994). Markov chain models a sequence of random variables $\left\{X_{k}, k=0,1,2 \ldots\right\}$ for which the Markovian property is held. Let $\left\{s_{1}, \ldots, s_{M}\right\}$ be a finite set of the possible mutuallyexclusive states of each $X_{k}$. The probability distribution over these states is represented by the vector $p\left(X_{\mathrm{k}}\right)$ :

$$
\begin{aligned}
& p\left(X_{k}\right)=\left[p\left(X_{k}=s_{1}\right), \ldots p\left(X_{k}=s_{m}\right), \ldots, p\left(X_{k}=s_{M}\right)\right] \\
& \text { with } \sum_{m=1}^{M} p\left(X_{k}=s_{m}\right)=1
\end{aligned}
$$

In this section, only systems having constant failure rates are considered. Homogenous Markov chains are used for this case because of the stationarity of transition probabilities. In such model, transition probabilities are time invariant and depend only on values states:

$$
\begin{aligned}
p_{i j} & =p\left(X_{k+1}=s_{i} \mid X_{k}=s_{j}\right) \\
& =p\left(X_{k+2}=s_{i} \mid X_{k+1}=s_{j}\right)
\end{aligned}
$$

In a homogeneous discrete-time $\mathrm{MC}$, the transition matrix $\mathbf{P}_{M C}$ between the states is defined from failure rate parameters. For instance let us consider a system with two states $\{1,2\}$, then:
$\mathbf{P}_{M C}=\left[\begin{array}{cc}1-p_{12} & p_{12} \\ 0 & 1\end{array}\right]$

Where $p_{12} \cong \lambda \Delta t: \lambda$ presents a constant failure rate and $\Delta t$ is the time interval. The probability $p_{12}$ can be interpreted as the probability that the component fails after the time $\Delta t$.

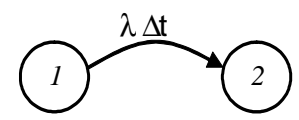

Fig. 1. Markov Chain for modelling deterioration model

Given an initial distribution over states $p\left(X_{0}\right)$, the probability distribution over states after $k$ stage $p\left(X_{k}\right)$ is obtained from the Chapman-Kolmogorov equation:

$$
p\left(X_{k}\right)=p\left(X_{0}\right) \prod_{i=0}^{K} P_{M C}
$$

Assuming that $i \in\{0 \ldots l\}$ represents the functioning states, system reliability is defined as:

$$
R(k)=\sum_{i \in\{0 \ldots l\}} p\left(X_{k}=s_{i}\right)
$$

The use of classic MC to model deterioration in systems needs to enumerate all possible states which lead sometimes to a huge transition matrix. Indeed, parameters quickly increase exponentially with respect to the number of reachable system states. Unfortunately, it is often infeasible to do this sort of exhaustive enumeration to determine the transition matrix.

\subsection{Time-dependent Markov process}

The basic assumption of discrete-time Markovian models is that the sojourn time in each state is geometrically distributed. Under this assumption, the failure rate of a component is considered as independent of time. Unfortunately, the geometric distribution is unsuitable for the majority of engineering problems because it does not reflect the fact that most component continuously deteriorates due to usage, wear or age.

To take it into account, the matrix defined in eq. 3 becomes time-dependant and the $\mathrm{MC}$ is not homogeneous anymore.

$\mathbf{P}_{M C}\left(X_{k} \mid X_{k-1}\right)=\left[\begin{array}{cc}1-p_{12}(k) & p_{12}(k) \\ 0 & 1\end{array}\right]$

One solution to model a time-dependent Markov process (e.g. Semi-Markov process (Limnios, 2001)) consists in an approximation of the initial process by an equivalent time-independent $\mathrm{MC}$. This transformation is usually provided by approaches such as the supplementary variables (Cox, 1955a) or equivalent rate methods as phase type distribution approximation (also called state space expansion or method of stages). The method consists in increasing the number of system states to model the dynamics or degradations (Cox, D.R., 1955b). Discrete phase type distributions (DPH) 
have been introduced and formalized by (Neuts, 1981). They are used in the numerical solution of non-markovian processes (Janssen et al., 2001), or closely related to physical observations.

According to the structure of the transition matrix $\mathbf{P}_{M C}$ and the initial probability vector $p\left(X_{0}\right)$, many (discrete or continuous) distributions can be approximated by the absorbing-time distribution of the MC (Fig. 1). Nevertheless, the fitting problem for the DPH class has received very little attention and precautions must be taken in order to know the accuracy and the restrictions of the method (Bobbio et al., 2004).

\begin{tabular}{|c|c|c|c|c|c|}
\hline \multirow[t]{3}{*}{$p\left(X_{0}\right)$} & & $\begin{array}{lll}1 & 0 & 0\end{array}$ & 0 & & \\
\hline & {$\left[1-p_{01}-p_{0 f}\right.$} & $p_{01}$ & 0 & $\ldots$ & $p_{0 f}$ \\
\hline & 0 & $1-p_{12}-p_{1 f}$ & $p_{12}$ & $\ldots$ & $p_{1 f}$ \\
\hline \multirow[t]{3}{*}{$\mathrm{P}_{M C}$} & $\ldots$ & $\ldots$ & $\ldots$ & $\ldots$ & $\ldots$ \\
\hline & 0 & 0 & 0 & $1-p_{i f}$ & $p_{i f}$ \\
\hline & 0 & 0 & 0 & $\ldots$ & 1 \\
\hline
\end{tabular}

$$
\begin{gathered}
\text { Graphical } \\
\text { Model }
\end{gathered}
$$

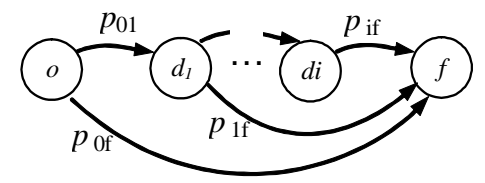

Fig. 2 - Canonical form CF3 (Bobbio et al., 2004).

\subsection{Non observable degradation model}

In the previous sections stochastic processes $X_{k}$ are supposed to be completely observable. In practice this is seldom the reality because the physical degradations of a component result in a change of its state which is observed only through a variation in the component functionality. Therefore a failure is an event impacting the component state which is modelled as a stochastic process $X_{k}$, nevertheless the only one phenomenon observed is the occurrence of failure modes in the component function which could be modelled as stochastic processes $Y_{k}$.

To model such processes with unobservable states or if the observation of this information is too expensive to be acquired, we propose to use a substitution variable $X_{k}$ to characterize the deterioration level and the component states. The random variables $X_{k}$ are assumed to be hidden stochastic process. In this case, model could be described as a Hidden Markov Model (HMM) where $p\left(Y_{k} \mid X_{k}\right)$ is the emission distribution. Usually used in speech recognition (Rabiner, 1989) or financial analysis, HMM is doubly stochastic process with an underlying stochastic process that is not observable (hidden). The process is observed through another set of stochastic processes that produces the sequence of observations. Therefore the general model of component degradation can then be formalized from HMM.

\subsection{Modelling under exogenous events}

Failure times are often considered as the only factor that can explain the reliability characteristics of the system. This may be insufficient (Singpurwalla, 1995). Operating and environmental conditions (e.g. humidity, temperature) may also influence the reliability of a system. All those factors are referred to as covariates or explanatory variables (Bagdonavicius, et al., 2001). The reliability characteristics of a system can be then explained in a better way by considering the effects of covariates. To take into account exogenous events, several models represent each situation according to an environmental context, and models become complex when the system is composed by $n$ components with several failure modes.

Markov Switching Models (MSM) are introduced to model this kind of stochastic process with exogenous events. These models are also viewed as conditional MC where the selection of transition distributions is dependent on exogenous variables. The MSM models the non-stationary due to abrupt changes in the functioning modes of the system (Bengio Y., 1999). A MSM represents the conditional distribution $p\left(X_{k} \mid U_{k}\right)$ given the input sequence $U_{k}=\left[u_{0}, u_{1}, \ldots, u_{k}\right]$ where $u_{k}$ represents the exogenous constraint with states $\left\{s_{1}^{u}, \ldots, s_{M}^{u}\right\}$.

The simulation of MSM is based on switching and initialisation procedure of MC depending on the state of the exogenous variable. It is unsuitable to obtain the analytic solution of this kind of hybrid differential system.

Moreover if it is a distribution over the states of the exogenous variable $U_{k}$ (input) which condition the solution, then we proposed the formalism of InputOutput HMM (IOHMM) to model the stochastic process. Let $Y_{k}$ represent the observations (output), $X_{k}$ the hidden variables and the external exogenous observable variables denoted as $U_{k}$ which induces several behaviours of the hidden process $X_{k}$. Moreover, the result of the hidden process is observed through the failure modes of the component defined as outputs $Y_{k}$. To model this complex stochastic process $p\left(Y_{k} \mid U_{k}, X_{k}\right)$, the formalism of Input-Output HMM (IOHMM) is well suitable.

\section{BAYESIAN NETWORK MODELLING}

The MC, HMM, MSM or IOHMM are well suited models in the reliability analysis of a complex entity or system of low dimension. However, within the framework of general complex systems, the combinatorial explosion of states makes these methods unmanageable. To overcome this problem, a method based on DBN formalism is presented in the next section.

\subsection{Bayesian Network notation}

Bayesian networks (BN) (Pearl, 1988) are directed acyclic graphs (DAG) representing a joint 
probability density function (Pdf). Nodes represent variables and directed arcs indicate causal relations between the connected nodes. According to dconnexion notion, the lack of arc between two nodes $X^{j}$ and $X^{l}$ (Fig. 3) means that the knowledge of $X^{l}$ is irrelevant to estimate the state value of $X^{j}$ when the state of $X^{i}$ is known. Whereas the existence of arc between two variables indicates a direct probability dependency between them as $X^{i}$ and $X^{j}$ in Fig. 2. For more details see (Jensen, 1996).

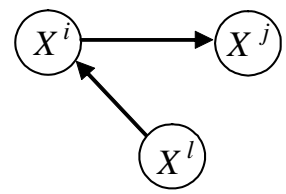

Fig. 3. Example of BN

Nodes which has not parent are described by a marginal distribution: unconditional probability. So they are characterized by a probability table (PT) composed of the class belonging probabilities $p\left(X^{i}\right)$ over the all possible states of $X^{i}$. Whereas, nodes having one parent at least (children nodes) are characterized by conditional distributions probabilities. So, Conditional Probabilities Tables (CPT) are associated with these type of nodes. PT and CPT together with the independence assumption defined by the graph, present a unique joint distribution over all the variables. Thus, it is possible to quantify probabilities for each variable conditioned on all the possible values the others variables in the graph.

\subsection{Dynamic Bayesian Network}

In this section, special case of BN named Dynamic Bayesian Networks (DBN) is proposed to model different types of time-dependent process.

DBN includes a temporal dimension managed by time-indexed random variables. The process is represented at time step $k$ by a node $X_{k}^{i}$ with a finite number of states $S_{X^{i}}:\left\{s_{1}^{X^{i}}, \ldots s_{M}^{X^{i}}\right\}$ and arcs represent dependencies across time points. The state space $\Omega$ is the cross product of the value spaces for individual states variables: $\Omega=\prod_{i=1}^{N} S_{X^{i}}$. Let $p\left(X_{k}^{i}\right)$ denotes the probability distribution over the variables states at time step $k$. Nodes correspond to the state variables can be partitioned into two sets: those corresponding to state of the variable at the current time step $(k)$, and those corresponding to state system at the following time step $(k+1)$. In this case, the variable is represented at successive times. In the DBN, shows in (Fig. 4), the temporal evolution is represented in two slices of time $k$ and $k+1$, so network is called by (Boyen and Koller, 1998) a two-Time slices Bayesian Network (2-TBN). Defining the transition-probabilities between the states of the variable at time step $k$ and time step $k+1$ leads to define CPT relatively to inter-time slices. So, given any $p\left(X_{k}^{i}\right)$ at time step $k$, the network induces a unique distribution $p\left(X_{k+1}^{i}\right)$ at time $k+1$ which is calculated by inference. Starting at time $k=0$ with the distribution $p\left(X_{0}^{i}\right)$, computation of $p\left(X_{T}^{i}\right)$ can be realized by iterative inferences.

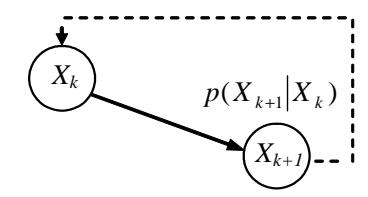

Fig. 4. A 2-TBN modelling MC with node $X_{k}$

A) Markov Chain model: The knowledge of the distribution probabilities and the CPT allows the calculation of the distribution probabilities at time $k+1$ by making inference. So, with this model, the future $(k+1)$ is conditionally independent of the past given the present $(k)$, which means that the CPT or $p\left(X_{k+1} \mid X_{k}\right)$ respects the Markov property (Kjaerulff, 1995). In the classical MC representation (\$2.1), parameters are calculated for all the possibilities of states variables leading to the state space $\Omega$, and the notion of independence between variables is unexploited. Contrary to this, DBN allows to take into account this consideration to represent joint Pdf in a factorized manner. In real world, the number of system states is very important, so a matrix representation is fastidious and usually unfeasible. The use of DBN modelling to analyse the deterioration system is more suitable to solve this problem, it permits to minimize considerably the number of parameters to stand for a joint Pdf (Weber and Jouffe, 2003).

B) N-order Markov model: For many stochastic processes, the first order MC is not sufficient to describe the deterioration model. Thus, usually we need to know more than one state in the past to predict the future. DBN offer an elegant way to model such hypothesis. The concept of 2-TBN can be generalized to higher order. Indeed, (N-1) order Markov property can be modelled by N-Time slices Bayesian Network (N-TBN) (Ben Salem, et al., 2004). In this case, the past is resumed in the $\mathrm{N}$ last times slices. Distribution probabilities are obtained by inference using CPT containing the state evolution over $\mathrm{N}$-time slices. The degree of the temporal regression is not bounded and it is possible to use any past instance information to determine probabilities distribution of the future.

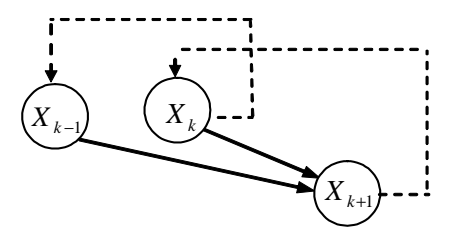

Fig. 5. A 3-TBN modelling second order MC.

Fig. 5 shows, how a second order MC can be modelled by a 3-TBN. Unfortunately, more the degree of the regression is important more complex 
is the CPT of the node $X_{k+1}$. Nevertheless this model is interesting to take into account the dynamic comportment of the behaviour of the distribution of the probability of $X_{k+1}$. This model provide an alternative solution to the problem described in $\S 2.2$.

C) semi-Markov model: with DBN the CPT models the dependence between the past and the future as the conditional probability $p\left(X_{k+1} \mid X_{k}\right)$. This CPT can be indexed by time then the CPT is not constant but involve according to $k$. In this way a $2-\mathrm{TBN}$ is able to simulate a semi Markov process.

D) Non observable degradation model: with DBN it is also possible to model non observable system through emission distribution $p\left(Y_{k} \mid X_{k}\right)$ with $Y_{k}$ the observed variable and $X_{k}$ the hidden variable. In this case when all the temporal nodes are independent, an exact inference algorithm is still used to simulate the model. A 2-TBN model of HMM is presented as a compact DBN form in (Fig.6). This framework provide a graphical modelling solution to the problem explained in $\S 2.3$.

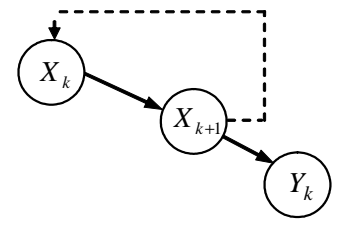

Fig. 6. A 2-TBN modelling MC with temporal node $X_{k}$ and exogenous observations $U_{k}$.

E) Exogenous event model: in DBN non homogeneous stochastic model is able to be computed with taking into account the effect of the exogenous events on systems (like accidentally rupture of a component). The formalism to realize this are MSM and IOHMM as it is presented in \$2.4. (Bengio and Frasconi, 1995) demonstrate how it is possible to model IOHMM with DBN. Weber et al. (2004), show an example of system reliability analysis formalised as MSM with DBN.

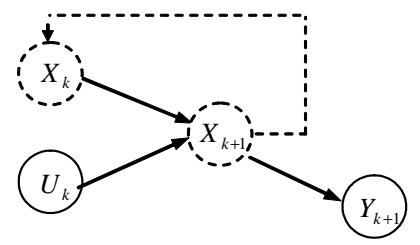

Fig. 7. A 2-TBN modelling IOHMM with temporal node $X_{k}$ and exogenous observations $U_{k}$ and $Y_{k+1}$.

Let's conceder the covariate as a (exogenous) discrete random variable $U_{k}$. This variable is modelled by a node containing information about exogenous events see (Fig. 7.). The node is realised in the current time slice $k$ and can be introduced as (hard) evidence or probability distributions observations. So, the introduction of the context made by the means of $U_{k}$ governs the behaviour of $X_{k}$. Observations at time $k$ condition the state probabilities distributions at time $k+1$, because of the CPT which are conditioned by the exogenous variables.

\subsection{DBN to model failure dependencies}

The reliability of component can be modelled as a DBN made of some nodes as presented in the \$3.2. Thus independent components in the system are modelled using DBN equivalent to independent MC/HMM/IOHMM. The power of this method is that the DBN allows to merge the MC, HMM or IOHMM through a factorised form (Murphy, 2002). This aggregation is made in the time slice $(k+1)$ with a method similar to the fault tree but without the limitation of independent elementary event and the variables can be described over $n$ states (several failures or degradations), Fig. 8.

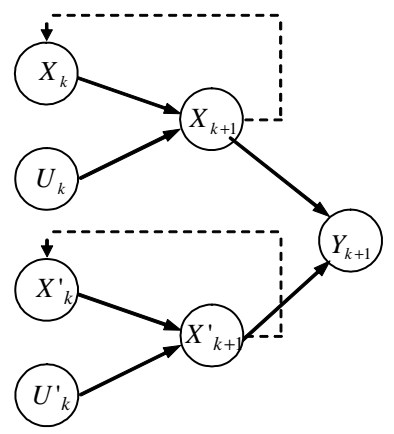

Fig. 8. DBN model of a system composed from tow independent stochastic processes.

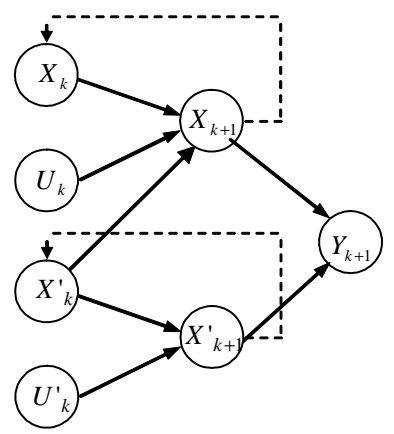

Fig. 9. DBN model of a system composed from dependent stochastic processes

One of the most difficult tasks in reliability analysis is to model a simultaneous failure processes. Two cases appear: interconnected failures processes or independent failure processes. Original solutions can be proposed based on DBN to model both cases. For the first case of system, the idea consist to develop $N$ models of (number of interconnected failure process) DBN evolving at the same time and connected by node representing the global system state. Fig. 8 gives an example of model with two independent failure processes modelled by DBN. This formalism leads to model global system reliability in spite of the independence between some of their failure subsystems (Weber and Jouffe 2006). Algorithms as junction tree are used to make exact inference. The time increment is carried out by setting the computed marginal probabilities of the node at time step $k+1$ as observations for its corresponding node in the previous time slice. Nevertheless, if dependences exist in the stochastic 
processes (Fig. 9.) then exact inference algorithms can not by employed because it leads to errors. Therefore approximate inference algorithms are proposed for instance the (Boyen and Koller, 1998) inference algorithm allows providing a bounded error or other approximate inference methods, as for example particle filtering (Koller and Lerner, 2000).

This approach represents a useful method to decrease the complexity of the model by grouping interconnected subsystems in a global deterioration model. So, through this method, it is possible to analyse in one time both of the entire system and its subsystems. For example, instead of including distinct technical components with dependencies, this structure could be represented as one entity. Under this method, the BN leads to model independent stochastic process as IOHMM then the global model of the system is simplified. If the dynamics of process are independent, then the exact inference method based on junction tree compute the simulation of the system reliability.

\section{CONCLUSION AND FURTHER WORK}

The proposed method, based on the Dynamic Bayesian Networks theory, easily allows designing DBN structures for the modelling of temporal evolution of complex systems. The correspondence between Markov Chains and DBN is presented and applied to the system reliability estimation.

The proposed method is a good solution to model the reliability of complex stochastic process. Indeed, the number of states needed to model a complex system with MC increases exponentially. As the DBNs representation is based on the modelling of process entities, the obtained model is more compact and readable than MC.

In future works, in order to achieve this modelling technique we have to define how the learning algorithms of $\mathrm{BN}$ can contribute to model the dynamics of the system reliability and how the parameters behaviour can be then modelled.

\section{REFERENCES}

Labeau, P. E., Smidts C. and Swaminathan S. (2000). Dynamic reliability: towards an integrated platform for probabilistic risk assessment Reliability Engineering and System Safety, 68(3), 219-254.

Ansell, J.I. and Phillips M.J. (1994). Practical methods for reliability data analysis. Oxford University Press Inc, ISBN 019853664 X, New York.

Bagdonavicius, V., Nikulin, M. (2001). Estimation in Degradation Models with Explanatory variables, Lifetime Data Analysis, 7, 85-103.

Barlow, RE. Prochan, F., (1965). Mathematical theory of Reliability. New York: Wiley.

Bengio, Y., (1999). Markovian models for sequential data. Neural Computing Surveys, 2, pp 129-162.

Ben Salem, A., Bouillaut, L., Aknin, P., Weber, P. (2004) Dynamic Bayesian Networks for classification of rail defects. IEEE 4th International Conference on Intelligent Systems
Design and Applications, Budapest, Hungary, August 26-28.

Bobbio, A., Portinale, L., Minichino M. and Ciancamerla E. (2001). Improving the analysis of dependable systems by mapping fault trees into Bayesian networks. Reliability Engineering and System Safety, 71, 249-260.

Boudali H., Dugan J. B. (2005). A discrete-time Bayesian network reliability modeling and analysis framework. Reliability Engineering and System Safety, 87(3):337-349, March.

Boyen, X. and Koller D. (1998) Tractable for Complex Stochastic Processes. In Proceedings of the 14th Annual Conference on Uncertainty in AI (UAI), Madison, Wisconsin, July 1998, 33-42.

Cox, D.R., (1955a) A Use of Complex Probabilities in the Theory of Stochastic Processes, In Proceedings of the Cambridge Philosophical Society 51, 313-319.

Cox, D.R. (1955b) The analysis of non-markovian stochastic processes by the inclusion of supplementary variables. In Proceedings of the Cambridge Philosophical Society, 51, 433-440.

Jensen, F.V. (1996) An introduction to Bayesian Networks, Springer-Verlag, 178, New York

Limnios, (2001) N. Semi-Markov processes and reliability, Boston, Birkhauser.

Koller, D. and Lerner, U. (2000) Sampling in Factored Dynamic Systems. Invited contribution to the book Sequential Monte Carlo Methods in Practice, A. Doucet, J.F.G. de Freitas, and N. Gordon, Eds., Springer-Verlag, pp 470-490.

Murphy, K. (2002) Dynamic Bayesian Networks: representation, inference and learning. These of the University of California, Berkley.

Neuts, M. F. (1981). Matrix-Geometric Solutions in Stochastic Models. John Hopkins University Press.

Pearl, J. (1988) Probabilistic Reasoning in intelligent systems- Network of Plausible Inference. Maurgan Kaufmann, San Francisco, CA.

Rabiner, L.R. (1989) A tutorial on hidden Markov models and selected applications in speech recognition. In Proceedings of the IEEE, Vol.77,No.2, February.

Singpurwalla, N.D, (1995) Survival in Dynamic Environments. Statistical Science, Vol. 10, No. 1, 86-103.

Torres-Toledano, J.G., Sucar, L.E. (2003). Bayesian Networks for Reliability Analysis of Complex Systems. Lecture Notes in Computer Science "Progress in AI - IBERAMIA'98, Lisbon, Portugal, Oct. 1998. Proceedings", SpringerVerlag Heidelberg, ISSN: 0302-9743, 1484, Jan 1998, 195- 206.

Weber, P., Jouffe, L. (2003). Reliability modelling with Dynamic Bayesian Networks. 5th IFAC Symposium on Fault Detection, Supervision and Safety of Technical Processes, Washington, D.C., USA, June 9-11.

Weber, P., Jouffe, L., (2006) Complex system reliability modelling with Dynamic Object Oriented Bayesian Networks (DOOBN). Reliability Engineering \& System Safety, 91(2), February, 149-162.

Weber, P., Munteanu, P., Jouffe, L. (2004). Dynamic Bayesian Networks modelling the dependability of systems with degradations and exogenous constraints. 11th IFAC Symposium on Information Control Problems in Manufacturing, Salvador-Bahia, Brazil, April 5-7. 